\title{
CLIDOPHORUS ET LA QUESTION DE LA DOUBLE PHILOSOPHIE
}

\author{
Pierre LURBE
}

Résumé: Dans Clidophorus, John Toland pose le problème de la relation entre philosophie ésotérique et philosophie exotérique : alors que la première nommée est la seule philosophie vraie, la seconde en est la traduction à l'usage du grand nombre. Il est en effet doublement nécessaire pour les philosophes de traduire la philosophie ésotérique en langage exotérique: il s'agit, d'une part, de se protéger, dans la mesure où la véritable philosophie contient un enseignement matérialiste radicalement incompatible avec les préjugés du vulgaire et des religions établies; et il s'agit, d'autre part, de donner à deviner la vérité sous des voiles suffisamment transparents pour que s'éveille la curiosité de disciples potentiels. La difficulté de l'entreprise tient donc à ce que la philosophie exotérique doit simultanément, et contradictoirement, permettre aux intelligences les plus fines d'acquérir un pressentiment de la vérité, sans mettre pour autant en cause les superstitions communément reçues. À cette aune, Toland juge que les fables des Anciens, forme antique de la philosophie exotérique, échouent dans la mesure où elles ne font qu'encourager la superstition; il suggère par contre implicitement que les métaphores dont Spinoza fait usage dans le Traité théologico-politique fournissent le modèle d'une philosophie exotérique qui traduise sans la trahir la philosophie ésotérique.

Mots clés : ésotérique, exotérique, fable, métaphore, Spinoza.

SUMmary : In Clidophorus, John Toland tackles the relationship between esoteric philosophy and exoteric philosophy: while the former is the only true philosophy, the latter is its translation in terms that are meant to be intelligible to the vast majority of men. It is necessary for philosophers to translate their esoteric philosophy into exoteric language for two reasons: on the one hand, as true philosophy consists in a materialist doctrine that is irreconcilable with the prejudices of ordinary men and established religions, philosophers must protect themselves from the fury of the mob and the priests; on the other hand, they must still manage to hint at the truth in sufficiently transparent terms to arouse the interest of potential disciples. Devising an adequate exoteric language is all the more difficult as it must simultaneously allow the finer minds to get an inkling of the truth, while at the same Revue de synthèse : $4^{e}$ S. $N^{\text {os }} 2-3$, avr.-sept. 1995, p. 379-398. 
time leaving the common prejudices undisturbed. In so far as the fables of the Ancients only foster superstition, Toland judges them as inadequate examples of exoteric language; conversely, he implicitly suggests that Spinoza's use of metaphors in his Tractatus theologico-politicus provides the model of an exoteric philosophy that is an adequate translation of the esoteric philosophy.

Keywords : esoteric, exoteric, fable, metaphor, Spinoza.

ZuSAMMENFASSUNG: In Clidophorus fragt Toland nach der Beziehung zwischen esoterischer und exoterischer Philosophie. Während die erste für ihn die einzig wahre Philosophie ist, hält er die zweite nur für deren Umsetzung für die breite Masse. Aus zwei Gründen ist es für die Philosophen erforderlich, die esoterische Philosophie in eine allgemeinverständliche - exoterische - Form zu bringen : einerseits handelt es sich dabei um eine Schutzmaßnahme, weil die wahre Philosophie eine materialistische Lehre enthält, die mit den allgemein verbreiteten Vorurteilen und den etablierten Religionen unvereinbar ist; andererseits mu $\beta$ man durch einen genügend durchsichtigen Schleier eine Ahnung der Wahrheit durchschimmern lassen, um die Neugier potentieller Anhänger zu erwecken. Die Schwierigkeit besteht darin, daß die exoterische Philosophie einen Widerspruch überwinden muß, indem sie es einsichtigeren Geistern ermöglicht, die Wahrheit $z u$ erahnen, ohne daß der allgemein verbreitete und überlieferte Aberglaube in Frage gestellt wird. Gemessen an diesem Anspruch sind für Toland die antiken Fabeln als früheste Form der exoterischen Philosophie gescheitert, weil sie ausschließlich den Aberglauben fördern. Er deutet dagegen an, daß die Metaphern, die Spinoza im Tractatus theologico-politicus benutzt, das Vorbild für eine exoterische Philosophie darstellen, die die esoterische Philosophie vermittelt, ohne sie zu verraten.

Stichwörter : esoterisch, exoterisch, Fabel, Metapher, Spinoza.

Pierre LURBE, né en 1956, est maître de conférences au Département d'anglais de l'université de Caen. Il est l'auteur d'une thèse et de nombreux articles consacrés au philosophe irlandais John Toland.

Adresse: Université de Caen Basse-Normandie, U.F.R. L.V.E., Département d'anglais, Esplanade de la Paix, 14032 Caen Cédex. 
En 1720, parvenu à l'âge de cinquante ans, John Toland publie presque simultanément deux ouvrages dans lesquels il nous propose ses réflexions sur les conditions de travail du philosophe, et spécifiquement sur les conditions dans lesquelles il peut produire, et surtout exprimer des idées, c'est-àdire les diffuser dans le cadre d'une société qui n'est pas spontanément favorable à l'activité philosophique. L'interrogation porte donc moins sur des contenus, que sur les conditions de production et de diffusion des idées philosophiques.

Ces deux cuvres, le Tetradymus et le Pantheisticon ${ }^{1}$, sont elles-mêmes publiées et diffusées dans des conditions fort différentes : la première, rédigée en anglais, est classiquement publiée par un éditeur ayant pignon sur rue, alors que la seconde, rédigée en latin à l'usage d'un public plus restreint, est publiée à compte d'auteur et diffusée, si l'on en croit la légende, sous le manteau. Malgré cette différence, Toland y traite explicitement, en termes identiques en général, ou parfois en termes complémentaires, de la question de la double philosophie ${ }^{2}$. Dans l'économie générale de ces deux livres, cette question est abordée, chaque fois, dans une sous-partie qui lui est spécifiquement consacrée : il s'agit, d'une part, de Clidophorus, l'un des quatre courts traités dont l'ensemble compose Tetradymus, et, d'autre part, de la troisième et dernière partie de Pantheisticon, qui a pour titre « De duplici Pantheistarum philosophia sequenda, atque de viri optimi $\&$ ornatissimi idea, dissertatiuncula ${ }^{3}$. Ces deux textes se font écho, puisque, comme le précise son sous-titre, Clidophorus traite

" of the exoteric and esoteric philosophy; that is, of the external and internal doctrine of the antients: the one open and public, accommodated to popular prejudices and the religions establish'd by law; the other private and secret, wherin, to the few capable and discrete, was taught the real truth stript of all disguises $\gg{ }^{4}$,

1. John Toland, Tetradymus, Londres, 1720 ; ID., Pantheisticon, Cosmopoli [sic] (en réalité Londres), 1720.

2. Cette question a en fait été abordée une première fois dans certaines des Letters to Serena, Londres, 1704, sans pourtant qu'il s'agisse de leur sujet principal : voir Letter II, p. 56-58; Letter III, p. 114 sq.

3. Voir la traduction anonyme du xvir siècle, repr. in Albert Lantone, Un Précurseur de la franc-maçonnerie. John Toland, 1670-1722, Paris, E. Nourry, 1927, p. 185 : « De la double philosophie que doivent suivre les panthéistes, et d'une petite dissertation qui donne l'idée d'un très honnête homme et d'un homme parfait. »

4. Voir Clidophorus, in Tetradymus, op. cit. supra n. 1, p. 61 (cité par la suite comme Clidophorus) : « de la philosophie exotérique et de la philosophie ésotérique; c'est-à-dire, de la doctrine externe et de la doctrine interne des Anciens; l'une ouverte et publique, accommodée aux préjugés populaires et aux religions établies par la loi; l'autre privée et secrète, où la vérité authentique, dépouillée de tout déguisement, était enseignée à ceux-là seulement qui étaient suffisamment capables et suffisamment discrets pour l'entendre. "Il s'agit bien de la 
cependant que la petite dissertation qui clôt le Pantheisticon précise :

«Sed vitio forsan vertetur Pantheistis, quod duplicem habeant doctrinam, externam scilicet vel popularem; vulgi praejudiciis, aut dogmatibus publice pro veris sancitis, utcunque accomodatam; \& internam vel philosophicam, rerum naturae, ac ipsi adeo veritati, penitus conformem : quodque secretam hanc Philosophiam, nudam \& integram, omni detracta larva \& ambage, non nisi (foribus occlusis) amicis spectatae probitatis \& prudentiae proponant "s.

Pour éviter toute ambiguité ultérieure, il faut noter d'emblée qu'au rebours de l'usage courant, pour lequel un discours dit «ésotérique » est obscur et impénétrable, le caractère ésotérique d'un discours ne se définit pas, selon Toland, par son obscurité, mais tout au contraire par sa simplicité et sa clarté, que conditionne le fait qu'il est réservé à un public averti et restreint; inversement et symétriquement, un discours exotérique n'est pas un discours clair et aisément intelligible, mais un discours à l'usage du grand nombre où la « vérité vraie » (the real truth) est en quelque sorte travestie par des figures qui la rendent très difficilement intelligible. La simplicité et la véracité du message d'une part, et l'étendue de l'auditoire d'autre part, sont donc inversement proportionnelles : à mesure que se restreint l'auditoire, cette simplicité et cette véracité augmentent, cependant qu'elles décroissent à mesure que l'auditoire s'accroît en nombre. Il en résulte ce paradoxe apparent que ce sont les propos exotériquement tenus qui sont obscurs et requièrent interprétation, alors que les propos ésotériques sont immédiatement intelligibles.

Les passages ci-dessus cités mettent en jeu le problème fondamental de la place de la philosophie dans la cité et de la relation entre les philosophes et le commun des hommes (ou vulgaire pour conserver le vocabulaire de l'époque), et posent directement trois questions, que je voudrais préalablement préciser, et qui seront successivement abordées dans le cours de la présente communication. Premièrement, puisque la philosophie ésotérique est posée par Toland comme originelle, et comme c'est elle qui doit faire l'objet d'une sorte de traduction sur le mode exotérique, il faut s'interroger d'abord sur la nature de cette philosophie ésotérique pour en préciser le

page de titre de Clidophorus, mais en même temps de la p. 61 de Tetradymus, dont la pagination est continue bien que l'ouvrage contienne quatre traités différents. Traduit par nos soins.

5. J. Toland, op. cit. supra n. 3, p. 240 : « Mais on fera peut-être un reproche aux panthéistes de ce qu'ils ont deux doctrines différentes, l'une exotérique ou populaire, accommodée aux préjugés et aux maximes reçues comme des lois saintes; l'autre ésotérique ou philosophique, entièrement conforme à la nature des choses et à la seule vérité; ou de ce qu'ils ne traitent cette philosophie nue, intègre et dépouillée de tout artifice et de toute obscurité, qu'à huis clos et avec des amis d'une prudence et d'une probité reconnue. » 
contenu; deuxièmement, il est nécessaire de décrire quels sont les obstacles qui s'opposent à la libre diffusion de la philosophie ésotérique, et qui rendent nécessaire le recours à une expression exotérique; troisièmement, il nous faudra préciser quelle forme, selon Toland, doit prendre la doctrine exotérique, ce qui ne sera possible que si l'on s'arrête d'abord sur la question des fonctions attendues de cette doctrine.

Toutefois, avant même de passer à notre premier point, une remarque préalable s'impose concernant le mode de traitement de la question par Toland dans Clidophorus : elle est traitée non pas tant de manière philosophique, que de manière historique. Conformément à une méthode d'approche déjà maintes fois utilisée par lui en d'autres endroits (comme les Letters to Serena, ou le Nazarenus de 1718, pour se borner à ces deux exemples), Toland veut écrire en historien, et prouver en remontant aussi loin que possible dans le passé l'extrême antiquité d'un procédé d'écriture, ou plus généralement de transmission de doctrines philosophiques, dont il nous dit en outre qu'il est toujours en usage de son temps : «I have more than once hinted, that the external and internal doctrine, are as much now in use as ever; tho the distinction is not so openly and professedly approv'd, as among the Antients ${ }^{6}$.

Aussi Clidophorus se présente-t-il au premier abord comme une laborieuse et fastidieuse compilation de références et de citations, tirées de Diogène Laërce, Clément d'Alexandrie, du De natura deorum de Cícéron, de Tertullien et d'autres auteurs antiques, et comme un passage en revue de philosophes ayant eu recours à la double doctrine, tels Pythagore, Aristote, Platon, ou les Académiciens. Lecture fastidieuse disions-nous, mais aussi quelque peu embrouillée dans la mesure où Toland n'adopte pas un plan strictement chronologique, mais revient, par exemple, à deux reprises sur l'exemple de Pythagore, dans deux contextes différents, de même qu'il revient à deux reprises sur Platon. Cette succession d'exemples donne beaucoup moins le sentiment d'une progression par démonstration, que d'une sorte de piétinement, d'une inlassable répétition de ce qui n'apparaît au fond que comme un même exemple toujours réitéré sous des formes à peine distinctes pour enfoncer le même clou et énoncer le même constat : «Wherfore Parmenides was unquestionably in the right, when he affirm'd that there are two sorts of Philosophy, the one according to truth, the other according to opinion ${ }^{7}$; ou bien encore, une dizaine de pages plus loin, «But why do I insist on particulars? since this distinction of exoterical and

6. Clidophorus, p. 94 : « J'ai plus d'une fois laissé entendre que la doctrine externe et la doctrine interne sont tout autant que jamais en usage, bien que cette distinction ne soit si ouvertement professée que chez les Anciens. "

7. Clidophorus, p. 69 : « De sorte que Parménide avait sans nul doute raison, quand il affirmait qu'il existe deux sortes de philosophie, l'une selon la vérité, l'autre selon l'opinion. » 
esoterical doctrines, was, as it were, the Catholic establishment of all nations $»^{8}$. On se bornera pour l'instant à noter que ce sentiment de monotonie et de répétition peut être le résultat d'un "art d'écrire " - pour reprendre l'expression fameuse de Leo Srauss ${ }^{9}$ — visant délibérément à brouiller le message, et qu'il ne doit pourtant pas nous empêcher d'apercevoir les différences, explicitement signalées, que Toland fait entre les philosophes qu'il cite : si tous, selon lui, recourent en effet à la double doctrine, ce n'est pas nécessairement tout à fait de la même manière.

De la philosophie ésotérique, nous avons déjà noté qu'elle s'adresse à un auditoire restreint de sages capables d'entendre la vérité sans fard. Mais il faut encore préciser ce que Toland entend par cette vérité que les hommes aspirent à connaître, et qui est simplement appelée la vérité; autrement dit, quel est l'objet de la philosophie ésotérique, et quelle est la vérité à laquelle elle donne accès?

L'objet propre de la philosophie ésotérique est constitué par la réflexion sur la nature de Dieu, du monde et de l'âme, par des questions métaphysiques en d'autres termes. La vérité sur ces questions, telle que Toland nous la donne à connaître dans les Letters to Serena, Pantheisticon, et même, par allusions, dans Clidophorus, est que la réalité dernière se résume à la seule matière, éternelle et incréée, et à ses vicissitudes, au jeu purement mécanique de causes involontaires et de facultés aveugles ${ }^{10}$, de sorte qu'il n'existe rien de plus excellent que l'univers ${ }^{11}$, et donc évidemment pas de dieu créateur distinct du monde. Soumis aux mêmes lois que le reste de l'univers, l'homme meurt corps et âme, si bien que la mort n'est pas à redouter, contrairement à ce que craignent les superstitieux qui y voient le plus grand des maux ${ }^{12}$. L'hypothèse qu'adopte ici Toland est qu'il existe une "philosophia perennis", ou une «prisca theologia», connue depuis la plus haute Antiquité, dont tous les véritables philosophes ont eu connaissance, et qui s'est transmise intacte à travers les siècles : $\mathrm{Pla}$ -

8. Clidophorus, p. 80 : « Mais à quoi bon insister sur des exemples? puisque cette distinction des doctrines exotériques et ésotériques était, pour ainsi dire, l'institution universelle de toutes les nations. 》

9. Nous nous bornons ici à reprendre l'expression utilisée, sans faire autrement référence au contenu que lui donne Leo STRauss, in La Persécution et l'Art d'écrire, trad. Olivier BERRICHON-SEDEYN, Paris, Presses-Pocket, 1989, par crainte, sans doute, d'être tellement hypnotisé par le caractère séduisant de la thèse qu'il expose dans son article célèbre sur la persécution et l'art d'écrire que notre lecture de Clidophorus en soit involontairement plus gauchie qu'enrichie. Mais Laurent JAFFro a fait la preuve que, en n'hésitant pas à se servir des outils que propose Leo Strauss, on pouvait lire Toland avec pertinence : voir son article, «L'art de lire Toland », dans le présent volume infra, p. 399-419).

10. Clidophorus, p. 90 : «involuntary causes and blind faculties. »

11. Clidophorus, p. 91 : « the Universe, than which those philosophers, says Augustin very rightly, judge nothing more excellent to exist. "

12. Clidophorus, p. 67 : " not to mention the last of all evils death, which at least is so in their opinion. » 
ton, Pythagore, mais encore Moïse partageaient au fond, s'il faut l'en croire, la même doctrine matérialiste et panthéiste. On ne peut manquer de souligner également ici combien cette philosophie, en dépit des nombreuses références que fait Toland au stoïcisme, évoque la sagesse épicurienne et son « quadruple remède ${ }^{13}$. De ce climat épicurien, le meilleur exemple est peut-être fourni par Pantheisticon, où les associés socratiques, comme les disciples d'Épicure, sont décrits mangeant et philosophant ensemble :

«Sed, ut uno verbo rem expediam, dantur proculdubio in plurimis locis Pantheistae non pauci : qui, aliorum more, suos sibi habent privatos Coetus \& Sodalitates ubi conviventur, \& quod suavissimum est condimenti genus, ubi desuper philosophentur ${ }^{14}$.

Venons-en maintenant aux raisons qu'ont les philosophes de recourir à la double doctrine. Raisons au pluriel, et non au singulier, car ce recours à cette sorte de double langage peut renvoyer à deux ordres de raisons très différents, voire antagonistes. Soit, en effet, les philosophes sont contraints à agir de la sorte par des raisons purement extérieures à leur activité de pensée et de réflexion, et qui prennent la forme conjointe de la contrainte légalement établie de se conformer à certains dogmes, et de la pression de l'opinion du commun des hommes, soit au contraire le recours à une double philosophie provient d'un choix délibéré, motivé par ce qu'ils comprennent de la nature même de la réflexion philosophique (ces deux ordres de raisons - les unes que l'on peut dire externes, les autres internes - n'étant d'ailleurs pas nécessairement totalement incompatibles).

La contrainte a donc deux visages : celui de la contrainte légale, et celui, plus redoutable encore, de l'opinion. Contrainte légale, d'abord, que celle qu'imposent les prêtres - terme générique s'appliquant à toutes les religions établies, les païennes comme la chrétienne -, par l'intermédiaire des pouvoirs séculiers, sous forme d'obligation statutaire de croire à certains dogmes sous peine de sanctions pénales pouvant aller jusqu'à la mort. Puisque Toland écrivait au tout début du xvmI siècle, il peut n'être pas inutile de rappeler ici que Thomas Aikenhead fut supplicié à Édimbourg en 1697 pour avoir mis en cause la Trinité et l'autorité de l'Écriture, cependant qu'en Angleterre même, une loi promulguée sous Guillaume d'Orange

13. «Il n'y a pas de dieu à craindre; la mort est en dehors de toute sensation; le bonheur est accessible; on peut supporter ", cité par Jean-François DuvERNoY, in L'Épicurisme et sa tradition antique, Paris, Bordas, 1990, p. 92.

14. Voir J. TOLAND, in op. cit. supra n. 3, p. 247 : « il y a dans plusieurs endroits beaucoup de panthéistes qui, selon la coutume des autres, ont leurs sociétés et leurs assemblées particulières, dans lesquelles ils mangent ensemble, et, ce qui est infiniment au-dessus, ils y philosophent ensemble. » 
en 1698 frappait d'incapacité civile et de peines de prison quiconque nierait, par écrit ou en paroles, l'une des personnes de la Trinité, ou déclarerait qu'il y avait plus d'un dieu. Cette menace de persécution doit toutefois être considérablement relativisée, puisque Toland n'en fut pas pour autant empêché, malgré des tentatives de la Convocation de l'Église d'Angleterre au tout début du siècle (1702), de continuer à professer par écrit des opinions très hétérodoxes, qui, si elles lui valurent des critiques (comme ce fut le cas pour les deux dernières des Letters to Serena, consacrées à une soidisant réfutation des idées de Spinoza), n'entraînèrent pourtant pas de poursuites contre lui.

Mais plus dangereuse encore que la contrainte légale, la contrainte de l'opinion : si les prêtres sont à redouter, ils le sont peut-être moins à tout prendre que le vulgaire, dont on ne peut mettre en cause les superstitions et les préjugés sans risquer, et parfois perdre, sa vie. L'un des quatre traités composant Tetradymus, et, de manière significative, celui-là même qui succède directement à Clidophorus, est consacré à la philosophe alexandrine Hypatie, mise à mort par une foule furieuse pour avoir professé ouvertement des doctrines païennes.

Ces deux formes de contrainte n'en font en réalité qu'une : il n'y a pas simple juxtaposition entre les prêtres d'un côté, le vulgaire de l'autre, mais relation fonctionnelle entre les deux. Pour comprendre la manière dont Toland conçoit cette relation, il faut repartir des considérations par lesquelles il commence Clidophorus.

Si l'on suit le début du texte, Toland décrit l'homme non pas tant comme capable de vérité, que comme désireux de l'acquérir, ce penchant ou cette appétence étant simultanément contrarié par deux facteurs : la paresse naturelle, qui le dissuade de se lancer dans les études difficiles qui sont nécessaires pour acquérir le vrai, et l'activité professionnelle, qui éloigne tout aussi nécessairement de la recherche de la vérité ${ }^{15}$. L'un et l'autre de ces facteurs apparaissent comme purement accidentels et ne semblent pas devoir mettre en cause une commune capacité, essentielle celle-là, à atteindre la connaissance du vrai. Mais, profitant de cette situation, poursuit Toland:

" some cunning persons thought they cou'd not better attain to authority over the rest (which draws riches after it of course) than by pretending to be masters of this same truth. Next they gave it out that they could impart it to others, without putting them to any labor, or diverting them from any business : and

15. Clidophorus, p. 63-64: «for men being observ'd to be naturally fond of truth, tho, thro laziness or occupation, few are capable to acquire the possession of it. 》 
as for a little expense, who would grudge to give a price out of his transitory pelf for the invaluable jewel of knowledge? " ${ }^{16}$.

La relation entre ce qui deviendra la caste sacerdotale et le vulgaire est présentée comme un marché, mais un marché de dupes : si le vulgaire paie, et paie très cher, pour entretenir les prêtres, il n'en obtient pourtant pas la vérité, mais des fables concoctées à plaisir pour le maintenir dans un état de sujétion. Dans ce récit simplifié, Toland prétend décrire à la fois les origines du pouvoir politique, et les conditions de son maintien. Les « habiles empiristes ${ }^{17}$ qu'il met en scène sont des législateurs et des fondateurs de cités (tels que Minos, Numa, Mahomet, ou même Moïse, pourtant qualifié de « législateur incomparable ${ }^{18}$ ) qui assoient leur pouvoir politique sur des croyances religieuses, relayées par une caste spécialisée, et qui visent toutes à susciter l'obéissance par la crainte des châtiments de l'au-delà. Ces croyances religieuses sont de pures et simples superstitions, c'est-à-dire, au sens strict, des croyances portant sur des domaines à propos desquels il est impossible d'acquérir la moindre connaissance par des moyens naturels : qui, en effet, est revenu de l'au-delà pour en décrire les tourments?

Les prêtres ont donc besoin de maintenir le reste des hommes dans l'ignorance pour pouvoir continuer à vivre à leurs dépens ${ }^{19}$, de sorte que cette ignorance même contribue à faire du vulgaire la propriété du clergé ${ }^{20}$, qui peut le manipuler à sa guise; ainsi sont créées les conditions qui rendent l'activité philosophique, libre recherche de la vérité, non seulement difficile, mais extrêmement dangereuse, et qui, en faisant de la propagation de la vérité une tâche impossible, rendent nécessaires, pour des raisons de prudence, l'adoption par les philosophes d'un langage ambigu et équivoque, ou en un mot d'une double doctrine:

«Hence no room was left for the propagating of truth, except at the expense of a man's life, or at least of his honour and employment, wherof numerous examples may be alledg'd ${ }^{21}$.

16. Clidophorus, p. 64 : « des hommes rusés s'avisèrent qu'il n'y avait pas de meilleur moyen pour asseoir leur autorité sur les autres (ce qui permet en outre de s'enrichir) que de prétendre posséder cette même vérité. Puis ils firent savoir qu'ils pouvaient la communiquer aux autres, sans que ces derniers aient à faire aucun effort, ni qu'ils soient distraits de leur travail : et qui rechignerait à donner un peu de son transitoire butin en échange de l'inestimable joyau de la connaissance? »

17. Clidophorus, p. 64 : « crafty empirics. »

18. Clidophorus, p. 65 : «incomparable Legislator. »

19. Clidophorus, p. 89 : «the ignorance of the laity is the revenue of the clergy. "

20. Clidophorus, p. 68, souligné par nous: Toland parle bien d' « ambitious Priests, supported by their property the mob ».

21. Clidophorus, p. 65 : «Ainsi il ne resta aucune place pour la propagation de la vérité, sinon à risquer sa vie, ou au moins son honneur et son gagne-pain, ce dont il existe de nombreux exemples. » 
«This must of necessity produce shiftings, ambiguities, equivocations, and hypocrisy in all its shapes; which will not merely be call'd, but actually esteem'd necessary cautions $" 22$.

C'est donc sous l'effet de la contrainte tout extérieure que leur imposent les prêtres et le vulgaire que les philosophes sont conduits à utiliser tantôt une doctrine exotérique, tantôt une doctrine ésotérique. On a là une description pour ainsi dire canonique du « complot des prêtres », qui a l'avantage de proposer, à l'origine de cette dualité de discours, une raison qui demeure extrinsèque à l'activité philosophique, ce qui peut laisser supposer que viendra un moment où cet écart entre philosophie ésotérique et philosophie exotérique n'aura plus de raison d'être, une fois suffisamment affaibli le pouvoir du clergé.

Mais l'hypothèse du « complot des prêtres » n'est pas la seule à être évoquée par Toland dans Clidophorus. Le recours à une double doctrine peut en effet aussi relever d'un choix délibéré, et non être l'effet d'une contrainte; Toland explique en effet que nombreux sont les philosophes qui pensaient que l'on ne devait exposer les vérités divines et les vérités naturelles que par des figures, c'est-à-dire au moyen d'un discours exotérique requérant interprétation ${ }^{23}$. L'obscurité de l'expression devient, pour le vulgaire, incitation au respect de vérités qui lui échappent, pour le sage, incitation à philosopher. Les philosophes peuvent donc estimer en toute liberté qu'un langage hermétique est le seul qui convienne à la saisie intellectuelle d'un objet de pensée particulier, auquel cas ce n'est pas seulement la catégorie de public visé, ni les conditions historiques de diffusion du discours philosophique, mais la nature de l'objet même à penser qui commande et conditionne l'emploi d'un certain type de langage. Il ne peut jamais $y$ avoir, dans cette hypothèse-ci, de réduction d'écart entre la doctrine ésotérique et la doctrine exotérique, qui persisteront même dans le cas où disparaîtrait le pouvoir clérical. Au constat de l'existence d'une contrainte extrinsèque obligeant le philosophe à la prudence et à la dissimulation, s'ajoute donc l'hypothèse d'une nécessité intrinsèque le conduisant à choisir une expression délibérément énigmatique.

Si Toland s'emploie à montrer par de multiples exemples historiques la validité du constat, il ne s'emploie pas moins à récuser l'hypothèse d'une adéquation entre l'objet de la philosophie et une expression figurée, posant

22. Clidophorus, p. 68 : « Ceci rend nécessairement ondoyant, ambigu, équivoque et hypocrite, de toutes les manières; ce que l'on ne se contentera pas de nommer des précautions nécessaires, mais que l'on tiendra bel et bien pour telles. »

23. Clidophorus, p. 80 : «And indeed, not to speak of designing men, many others were persuaded, that both natural and divine things might best be explain'd by figures. » 
ainsi une différence entre un usage légitime de la double philosophie, et un usage illégitime et même dangereux.

La traduction en mode exotérique de l'enseignement philosophique ésotérique apparaît comme une tâche d'autant plus difficile que cette philosophie, matérialiste et mécaniste, heurte de front les préjugés populaires sur lesquels s'appuient, et que renforcent en retour, les religions établies : croyance en la création du monde par un dieu bienveillant, doué d'intelligence et de volonté, croyance en la résurrection, croyance en l'immortalité de l'âme, croyance aux châtiments et aux récompenses dans le monde à venir, bref, un ensemble de croyances conditionnées par une représentation anthropomorphique de l'univers.

À l'écart, immense, qui sépare la philosophie ésotérique des opinions vulgaires et des préjugés communs, qui sont en position antithétique, on mesure la difficulté toute particulière, et extrême, que revêt pour le philosophe la tâche d'«accommoder » sa pensée aux opinions vulgaires. On peut donc se demander, d'une part, quelle nécessité il y a à le faire et, d'autre part, de quelle manière le faire, sans passer de l'«accommodement" à la pure et simple dénaturation de la «vérité vraie» ( real truth»)?

La nécessité de le faire est évidente, puisque si le philosophe tient à la perpétuation de la doctrine dont il a lui-même hérité, il lui faut bien se trouver des disciples, qu'il ne peut atteindre, pour les raisons ci-dessus évoquées, que par le biais d'un discours exotérique. Puisqu'en outre, ces disciples potentiels se trouvent initialement mêlés au commun des hommes, il faut définir une forme de discours qui, tout en permettant aux intelligences les plus déliées d'acquérir comme un pressentiment de la vérité, ne soit pourtant pas de nature à perturber un ordre social somme toute bien assis. Il faut donc tenir un discours qui réponde simultanément à trois critères : il doit être à la fois intelligible à tous, non contradictoire avec l'ordre, et permettre d'accéder « à quelque substance métaphysico-religieuse positive et à un semblant de vérité ${ }^{24}$.

La formulation de la doctrine exotérique exige que soit défini un bon usage des figures, dont Toland décrit ainsi le principe : « Not plainly to say and profess all you think, or to do it by circumlocution and figures, is one thing: but tis quite another thing, to speak positively against your own judgment, or against the truth in any figure of speech whatever ${ }^{25}$.

24. Formule empruntée à Yrmiyahu Yovel, Spinoza et autres hérétiques, Paris, Seuil, 1991, p. 200.

25. Clidophorus, p. 81 : «Ne pas dire et professer crûment tout ce que l'on pense, ou le faire au moyen de circonlocutions et de figures, est une chose; mais c'est tout autre chose de parler positivement contre son propre jugement, ou contre la vérité, en recourant à quelque figure du discours que ce soit." 
De manière toute négative, cette phrase pose une distinction entre le discours exotérique licite, et celui qui ne l'est pas, c'est-à-dire entre celui qui repose sur des figures acceptables, et d'autres qui ne le sont pas. Si nous suivons le conseil de lecture que nous donne Toland lui-même, il ne devrait pas être trop difficile de trouver la " clé » (et Clidophorus n'est-il pas, justement, le porteur de clé ?) qui doit permettre de distinguer les doctrines exotériques acceptables, de celles qui ne le sont pas : "The readers wanted a key, that might open'em a passage into his secret meaning : and such a key, that I may hint it en passant, is to be, for the most part, borrow'd by the skilful from the writers themselves ${ }^{26}$.

Cette clé ne se trouve pas très loin; il suffit de la chercher dans la succession d'exemples historiques donnés par Toland d'emploi par les philosophes de la double doctrine, dont nous soulignions pour commencer le caractère apparemment répétitif, puisque de cette succession ne découlait qu'un même constat, indéfiniment répété, celui du caractère universel du recours à la double doctrine.

Mais parmi les philosophes, on peut faire une distinction entre deux groupes, et deux types d'utilisation des figures : ceux qui emploient des fables, mode de discours que Toland estime illicite et même dangereux, et ceux qui recourent aux métaphores, seules capables selon lui de transmettre quelque sentiment de la vérité des choses.

Par fable, Toland comprend tout récit où figurent des dieux anthropomorphes, et qui comme tels sont représentés comme soumis à des passions humaines. Ce qui à ses yeux est grave, c'est que les fables, notamment les fables homériques, sont la traduction en termes exotériques inadéquats d'une doctrine ésotérique qui, pour sa part, peut être tout à fait conforme à la vérité des choses. Certes, Homère savait parfaitement que ses fables recouvraient une doctrine naturaliste, et que ses dieux désignaient non des êtres réels et doués de volonté et de passions, mais le jeu des causes naturelles ${ }^{27}$, mais comment ne pas voir que les fables qui servaient exotériquement de voile à ses doctrines ésotériques, en relatant les conflits et les guerres entre les dieux, leurs viols, leurs adultères, leurs massacres, leurs mensonges ${ }^{28}$, contrevenaient aux trois critères plus haut énoncés ? Il s'agit en effet, et dès l'abord, de récits inintelligibles; même si l'on admet que la clé de leur signification peut à la rigueur être fournie par une interprétation allégorique à la manière des philosophes antiques, cette clé est pourtant fort

26. Clidophorus, p. 76: «Les lecteurs voulaient une clé qui leur permît d'accéder à la signification secrète de ses propos : et cette clé, je le suggère en passant, doit être prise par les habiles essentiellement chez les auteurs eux-mêmes. "

27. Clidophorus, p. 82 : «That all his gods are pure natural causes, qualities, incidents, and events, are proved in this very life. »

28. Clidophorus, p. 86: «all those fables concerning the contentions and warrs of the gods, their rapes, adulteries, slaughters, lies, thefts, distresses. » 
incertaine : car quel modèle d'interprétation choisir? L'allégorie physique, à la manière stoïcienne, l'allégorie philosophique, à la manière platonicienne, l'allégorie morale des néo-platoniciens, ou bien encore l'allégorie historique d'Evhémère? En outre, et par voie de conséquence, les fables n'offrent l'accès à aucun contenu métaphysico-religieux, ni à aucun semblant de vérité; enfin, il n'est pas certain, bien au contraire, qu'elles ne soient pas de nature à troubler le bon ordre et la paix de la cité.

Toland ne trouve donc aucun argument qui justifie le recours aux fables, qu'il soit d'ordre extrinsèque (la nécessité de la dissimulation), ou intrinsèque (l'idée que ce serait une forme adéquate à son objet). Ni les lettrés ni le commun des hommes n'y trouvent leur compte, à aucun des titres cidessus mentionnés.

En effet, les premiers nommés n'ont nul besoin de ce soi-disant aiguillon pour exciter leur intelligence, qui sera mieux employée à s'occuper de choses intelligibles et utiles, plutôt qu'à chercher en vain une hypothétique clé allégorique :

"To say that fables will quicken our diligence, is the same plea with that for mysteries; as if time lay heavily on our hands, and that we had no other occupation but criticism ${ }^{29}$.

«[...] and as for the learned, 'tis a jest to talk of exercising their diligence; when time can never fail them in imploying their industry about what is intelligible and useful $»^{30}$.

Quant aux seconds, bien loin de seulement soupçonner - ce que les lettrés pouvaient encore faire - que les fables puissent avoir un contenu intelligible, ils s'arrêtent à leur aspect sensible et prennent l'image pour la réalité, si bien qu'ils tombent dans l'idolâtrie, ou plutôt que leur idolâtrie se trouve comme renforcée et redoublée, en même temps qu'ils s'enfoncent dans une immoralité que les excès des dieux fabuleux justifient à leurs yeux :

«for men will never think that to be a vice or imperfection in themselves, which is a virtue or perfection in the divinity [...] but to the vulgar they seemed so many real persons and stories that had certainly happen'd; as the reverence of objects, excited in the learned by images, terminates among the unlearned in the images themselves ${ }^{33}$.

29. Clidophorus, p. 87 : «Dire des fables qu'elles aiguiseront notre diligence, est la même défense que l'on fait des mystères: comme si nous disposions de trop de temps, et que nous n'ayons rien d'autre à faire que nous livrer à l'étude critique. »

30. Clidophorus, p. 81 : « quant aux lettrés, c'est une plaisanterie de parler d'exercer leur diligence; alors que le temps ne fait pas défaut pour qu'ils appliquent leur industrie à ce qui est utile et intelligible. »

31. Clidophorus, p. 86 : « car les hommes ne tiendront jamais pour vice ou imperfection chez eux, ce qui est vertu ou perfection dans la divinité [...] mais aux yeux du vulgaire, cela semblait être autant de personnes réelles, et d'histoires réelles, qui avaient certainement 
Le paradoxe qui se fait jour ici pourrait être formulé de la manière suivante : en recourant aux fables, les philosophes produisent le même type de discours que ceux que le clergé, dont ils prétendent pourtant se distinguer, invente pour inciter les hommes à l'obéissance par la crainte des châtiments de l'au-delà; de sorte que, comme l'écrit Cicéron, les fables sont « "if not the parents" »- qui seraient plutôt les prêtres -, « "yet certainly the fosterers of superstition" ${ }^{32}$, et que les philosophes contribuent ainsi à redoubler la superstition, dont ils devaient pourtant aider les hommes à se défaire.

Toute l'antique théologie repose sur l'emploi de fables, utilisées par les premiers législateurs des cités pour imposer l'ordre aux hommes déraisonnables. Et Toland de citer Strabon :

"Wherfore Jupiter's thunderbolt, Minerva's shield, Neptune's trident, the torches and serpents of the Furies, and those spears which are reckon'd the arms of the gods, are all fables, as well as the whole antient theology: for these things were receiv'd by legislators, as so many bullbeggars, wherby to keep in order the silly part of mankind ${ }^{33}$.

L'argument est connu, et repris par de nombreux philosophes antiques, qui consiste à dire la superstition nécessaire à l'ordre; comme le dit Timée de Locres, si l'on ne peut gouverner l'esprit des hommes par de vraies raisons, il faut le faire par de fausses raisons. Serait-ce à dire que, si les fables ne sont pas justifiables pour des raisons spéculatives, puisqu'elles entravent plutôt qu'elles ne facilitent l'accès à la vérité des choses, elles le sont au moins par des raisons d'ordre politique, en tant qu'auxiliaires du maintien de l'ordre? Cette justification, nous l'avons déjà signalé, n'est pas retenue par Toland. Contre la superstition, il esquisse, plus qu'il ne développe, deux arguments complémentaires.

D'une part, quand bien même il serait vrai que la superstition ait pu produire le résultat requis, c'est-à-dire maintenir le vulgaire dans l'obéissance, il reste que ses effets sont statistiquement plus souvent néfastes que bénéfiques pour la vie des cités:

« But granting that superstition had at any time prov'd beneficial to the public, yet at other times without number, and in things of incomparably greater

existé; car la révérence que les images suscitent, et qui chez les lettrés se porte sur les objets qu'elles signifient, s'arrêtent aux images mêmes chez les ignorants. 》

32. Clidophorus, p. 82 : " "sinon les parents, du moins les nourrices de la superstition"."

33. Clidophorus, p. 82 : «De sorte que la foudre de Jupiter, le bouclier de Minerve, le trident de Neptune, les torches et les serpents des furies, et ces lances que l'on tient pour les armes des dieux, ne sont que fables, ainsi que toute l'antique théologie : car ces choses étaient reçues par les législateurs comme autant d'épouvantails grâce auxquels maintenir les sots dans l'obéissance. " 
importance, it will be found detrimental, destructive, and utterly pernicious; nor advantageous to any, excepting priests or princes, who dextrously turn it to their own interest, tho even these are not always able to direct it at their pleasure. It does not onely every where disturb private society and concord, and somtimes bring its sacred and soverain managers to the last extremities; but too often disorders, or quite overturns, most flourishing governments, which the histories of all ages and nations make evident by infinite examples ${ }^{34}$.

D'autre part, la superstition plonge l'individu qui en est, littéralement, affecté, dans une crainte perpétuelle : «As for the particular persons blasted with this contagious air, it never leaves'em a moment's tranquillity, waking or dreaming, in occurrences of life, or at the point of death ${ }^{35}$.

On est ici bien loin de l'idéal du sage, délivré de toute peur superstitieuse, qui constitue l'idéal de Toland; il utilisait volontiers, en effet, le pseudonyme d' «adeisidaemon », l'homme libéré de toute superstition qui ne redoute plus ni les dieux, ni la mort, et dont la devise pourrait être les vers de Virgile si souvent cités dans son œuvre : «Felix qui potuit rerum cognoscere causas,/ Atque metus omnes et inexorabile fatum/ Subjecit pedibus, strepitumque Acherontis avari! ${ }^{36}$.

À l'inverse de la fable, qui ne peut fournir un langage exotérique convenable, les symboles, et les métaphores, entre lesquelles Toland ne marque pas de différence, sont décrits par lui comme devant être les figures fondamentales de la philosophie exotérique. Mieux même, la métaphore a selon lui, si l'on peut dire, un véritable statut de «figure-pont», en ce sens qu'elle est simultanément ce qui permet de concevoir un objet de pensée, et d'en transmettre la connaissance aux illettrés eux-mêmes :

"In conceiving, explaining, and declaring the divine nature and attributes, I readily own that symbols and metaphors are not onely apt and useful, but the last of them even absolutely necessary. Nor, in truth, can any ideas of them be

34. Clidophorus, p. 82-83 : « Mais, en admettant même qu'il ait pu arriver que la superstition contribue au bien commun, en d'autres occasions, celles-là innombrables, et pour des choses incomparablement plus importantes, on s'apercevra qu'elle est nocive, destructrice, et totalement pernicieuse; et qu'elle ne comporte d'avantages pour personne, si ce n'est les prêtres et les princes, qui l'utilisent habilement à leur propre avantage, bien qu'ils ne parviennent pas toujours à la manipuler à leur guise. Non seulement elle perturbe partout la concorde et l'amitié privées, et conduit parfois aux dernières extrémités ses chefs souverains et sacrés, mais trop souvent encore elle plonge dans le désordre, ou renverse complètement, les gouvernements les plus florissants, comme l'atteste, par des exemples infinis, l'histoire de tous les temps et de toutes les nations. 》

35. Clidophorus, p. 83 : «Quant aux personnes infectées par cet air contagieux, elle ne leur laisse jamais un instant de répit, qu'ils veillent ou qu'ils rêvent, dans les circonstances de la vie, ou à l'instant de mourir. "

36. Entre autres endroits, ces vers sont cités in Letters to Serena, op. cit. supra n. 2, p. 61. 
otherwise imparted to the illiterate, of which this is not the place to render a reason ${ }^{37}$.

Toland semble voir là une figure dans et par laquelle pourrait s'abolir l'écart entre la doctrine ésotérique et la doctrine exotérique : alors que, de la fable à la réalité qu'elle voile, il y a une solution de continuité qui requiert l'interprétation allégorique et tous les risques d'erreur et de fourvoiement qu'elle entraîne, la métaphore permettrait un passage aisé et insensible d'un pôle à l'autre. Mais le conditionnel est ici de rigueur, car de ces métaphores, Toland ne fournit justement pas d'exemple dans Clidophorus. Dans la mesure où ces métaphores sont censées être intelligibles aux illettrés d'abord, et leur permettre d'accéder à ce semblant de vérité que nous évoquions plus haut, il ne peut semble-t-il guère s'agir des métaphores qu'emploie Toland ailleurs dans son œuvre, comme par exemple, dans les Letters to Serena, où l'univers est décrit sous la forme d'une sorte de grand être vivant dont les fonctions vitales (nutrition, digestion, évacuation) représentent les infinies vicissitudes de la matière éternelle : induisant des rêveries anthropomorphes, ou zoomorphes, sur la nature de l'univers, ces images risquent plutôt de laisser le vulgaire empêtré dans leur matérialité même. Nous laisserons en suspens la question de la nature possible des métaphores que Toland avait en vue, pour y revenir ensuite par un autre biais, et formuler une hypothèse à leur propos.

Mais il est possible de reconsidérer certains des thèmes abordés ici sous un angle quelque peu différent, à partir de la phrase énoncée dans la préface à l'ensemble de Tetradymus, où Toland précise quelle est l'intention sous-jacente à Clidophorus, quelle en est, en somme, la clé :

«The consideration of these things, sufficiently express'd in the work itself, induc'd me to publish Clidophorus : that they of our modern times may, in the history of former ages, behold their own pictures drawn to the life; and learn the causes as well as the cure of their distemper, yet without expecting thanks and reward for the physician ${ }^{38}$.

Dans ce passage, Toland signale explicitement que Clidophorus n'est pas simplement un ouvrage d'érudition historique, puisque ses contempo-

37. Clidophorus, p. 88 : « Pour concevoir, expliquer et énoncer la nature divine et les attributs divins, je reconnais volontiers que les symboles et les métaphores sont non seulement pertinents et utiles, mais que ces dernières sont même absolument nécessaires; et je soutiens qu'en vérité il n'est pas d'autre moyen d'en communiquer quelque idée aux illettrés, ce dont je ne peux rendre raison ici. »

38. Tetradymus, op. cit. supra n. 1, préf., p. VII : « La considération de ces choses, suffisamment exprimée dans l'ouvrage lui-même, m'a poussé à publier Clidophorus, afin que ceux qui vivent aujourd'hui puissent, dans l'histoire des époques passées, considérer leur vivant portrait, et apprendre par là les causes comme le remède de leur maladie, sans pourtant que le médecin en attende remerciements et récompense. » 
rains sont censés s'y voir comme en un miroir, et en tirer des leçons à leur propre usage. Mais qu'est-ce qui, au juste, parmi les thèmes développés dans Clidophorus, peut trouver une application contemporaine?

On voit mal, au premier abord, ce que les longs développements contre les fables peuvent avoir à dire à des hommes qui vivent au début du $\mathrm{XVIII}^{\mathrm{e}}$ siècle : après tout, ce mode d'écriture exotérique n'est plus en usage; à l'époque moderne, il n'existe plus, de toute manière, de philosophe qui s'exprime ainsi. Aussi le propos de Toland ne commence-t-il à s'éclairer que si on le prend au mot, lorsque au détour d'un développement déjà signalé plus haut, il affirme l'existence d'un lien entre les fables et les mystères, c'est-à-dire les mystères de la religion chrétienne : «To say that fables will quicken our diligence, is the same plea with that for mysteries; as if time lay heavily on our hands, and that we had no other occupation but criticism ${ }^{39}$.

Ce ne sont donc pas tant les fables païennes qui sont visées, que les mystères chrétiens (comme la transsubstantiation) à travers elles. On retrouve en fait ici l'idée implicite d'une continuité entre paganisme et catholicisme, thèse classique dans la polémique protestante depuis au moins le début du $\mathrm{XVII}^{\mathrm{e}}$ siècle, et dont Clidophorus représenterait une reprise tardive, quelque peu modifiée, mais encore parfaitement reconnaissable et explicite. Ainsi, les Pères de l'Église sont décrits comme responsables de la perversion du christianisme primitif, dont ils auraient corrompu la simplicité en inventant des mystères qui réinstallent le paganisme au cœur même du christianisme :

« [...] I am downright ashamed of those Fathers, who made such ordinary actions as eating bread, drinking wine, and dipping in water, or washing with it, to pass for tremendous and inutterable mysteries. Very intelligible and apposite figures we grant'em to be, very significative of the things they represent and exhibit; but containing nothing terrible or abstruse, much less inutterable or inconceivable. Nevertheless, what those Fathers onely feign'd to be mysteries, that they might in nothing come short of the Heathenism they had quitted; their successors took care shou'd become unintelligible to some purpose, and be tremendous in more senses than one, or in any sense except that of the Gospel ${ }^{40}$.

39. Voir supra n. 29.

40. Clidophorus, p. 79 : «J'ai grande honte de ces Pères, qui ont fait passer pour d'immenses et ineffables mystères des actions si ordinaires que celles de manger du pain, boire du vin, se tremper dans l'eau, ou s'y laver. Qu'il s'agisse de figures très intelligibles et très à-propos, qui signifient très bien les choses qu'elles représentent et manifestent, c'est ce que je ne contesterai point; mais elles ne contiennent rien d'effrayant ni d'abstrus, et moins encore d'ineffable ou d'inconcevable. Néanmoins, ces mystères que ces Pères n'ont fait que feindre, afin de ne le céder en rien à ce paganisme qu'ils avaient quitté, leurs successeurs prirent grand soin de les rendre inintelligibles à dessein, et immenses en plus d'un sens, ou dans tous les sens possibles, sauf dans celui de l'Évangile. » 
De manière révélatrice, Toland mentionne Numa Pompilius dès les toutes premières pages de Clidophorus, parmi les premiers législateurs s'attachant l'obéissance du vulgaire par l'invention de fables adéquates; or, Numa Pompilius est un exemple classique entre tous démontrant aux yeux des polémistes protestants la filiation entre le paganisme et le catholicisme. Pour citer Pierre Mussard : « Les Evesques de Rome, au changement qu'ils ont apporté en la religion, ont eu devant les yeux le patron que Numa Pompilius et les autres instituteurs du Paganisme en avoient tiré ${ }^{41}$.

Clidophorus fonctionne donc sur une homologie sous-jacente, et non totalement explicitée, entre religion chrétienne et paganisme antique : ici, comme là, on aurait à l'origine une doctrine, religieuse ou philosophique, parfaitement simple et claire, déformée ensuite dans un langage exotérique inintelligible (fables ou mystères), qu'il faudrait remplacer par un langage exotérique intelligible donnant la possibilité de remonter à la vérité première.

Il faut, certes, reconnaître ce que cette épure a de simplificateur par rapport à un texte plus complexe et ambigu, dans lequel, pour s'en tenir à cet exemple, les personnages antiques, comme Pythagore, sont simultanément les détenteurs d'une sagesse à forme religieuse qui en fait les maîtres de la vérité, et les auteurs de la superstition, ce en quoi leur rôle n'est guère éloigné de celui de ce clergé que Toland critique si âprement par ailleurs.

Mais elle permet de mieux préciser ce que doit être la double fonction d'un langage exotérique à l'usage des contemporains de Toland. Puisque la superstition est indéracinable de l'esprit humain, et la première des Letters to Serena montre suffisamment qu'on ne peut la réduire au seul effet d'un complot clérical :

«At cum superstitio semper eadem sit vigore, etsi rigore aliquando diversa; cumque nemo sapiens eam penitus ex omnium animis evellere, quod nullo pacto fieri potest, incassum tentaverit : faciet tamen pro viribus, quod unice faciendum restat; ut, dentibus evulsis \& resectis unguibus, non ad lubitum quaquaversum noceat hoc monstrorum omnium pessimum ac perniciosissimum ${ }^{42}$.

41. Pierte Mussard, Les Conformitez des ceremonies modernes avec les anciennes. Où il est prouvé par des autoritez incontestables que les ceremonies de l'Église romaine sont empruntées des Payens, Leyde, 1667, p. 2, cité par Bernard DOMPNIER, «L'Église romaine, conservatoire des religions antiques. La critique protestante du culte des saints et des images au $\mathrm{XVI}^{e}$ siècle $\%$, in Les Religions du paganisme antique dans l'Europe chrétienne, xvf'xvif' siècle, Paris, Presses de l'université de Paris-Sorbonne, 1988, p. 53.

42. Voir J. Toland, in op. cit. supra n. 3, p. 241 : « Un homme sage ne fera jamais de vains efforts pour la déraciner entièrement de l'esprit humain, ce qui est absolument impossible; cependant, il fera tout ce qu'il pourra pour réussir à la seule chose qui lui reste à faire, qui est d'arracher les dents et de couper les ongles à ce monstre, le plus pernicieux de tous, pour le mettre hors d'état de nuire aux uns et aux autres, suivant sa fantaisie. » 
Pour réaliser ce programme, deux voies complémentaires se présentent : instaurer la liberté d'expression des opinions spéculatives, et parallèlement, mettre en place un langage religieux à l'usage des humbles, dont la formulation prenne appui sur les préjugés communs, qu'il ne faut pas heurter de front pour maintenir la paix de la cité, sans que le contenu en soit contradictoire pour autant avec la vérité philosophique; telle est la double fonction précise que Toland assigne apparemment aux métaphores dont il parle.

Or, il existe au moins un exemple récent de langage religieux à l'usage des humbles qui répond à cette double fonction : celui des « croyances fondamentales que l'Écriture universelle a pour objet d'établir ${ }^{43}$ que Spinoza énumère au chapitre xIv du Traité théologico-politique : 1) qu'il existe un dieu; 2) qu'il est unique; 3) qu'il est omniprésent; 4) qu'il a sur toutes choses droit et pouvoir suprême; 5) que le culte à lui rendre consiste dans la seule justice et la charité ; 6) que tous ceux qui suivront cette règle de vie seront sauvés; 7) que ce dieu pardonne leurs péchés aux repentants. Dans le sixième de ces articles, pour se borner à ce seul exemple ${ }^{44}$, la notion de salut est à la fois conforme aux superstitions vulgaires, et métaphoriquement conforme à la vérité, puisque le salut peut aussi signifier « la connaissance du troisième genre, associée à l'amour intellectuel de la nature-Dieu ${ }^{45}$. Pour prendre un autre exemple, tiré du chapitre III du Traité théologico-politique, et plus probant encore dans la mesure où c'est Spinoza lui-même qui parle :

«Par gouvernement de Dieu j'entends l'ordre fixe et immuable de la nature, autrement dit l'enchaînement des choses naturelles; nous avons dit plus haut en effet et montré ailleurs que les lois universelles de la nature suivant quoi tout se fait et tout est déterminé, ne sont pas autre chose que les décrets éternels de Dieu qui enveloppent toujours une vérité et une nécessité éternelles; que nous disions donc que tout se fait suivant les lois de la nature ou s'ordonne par le décret ou le gouvernement de Dieu, cela revient au même " ${ }^{46}$.

Il y a sans doute quelque chose d'abusif à interpréter les silences d'un texte; pourtant, en guise de conclusion, nous nous arrêterons à l'hypothèse que, en évoquant la nécessité pour le philosophe de recourir aux métaphores, Toland avait en tête le modèle fourni par Spinoza en la matière. Hypothèse qui vaut ce qu'elle vaut, mais qui n'est sûrement pas

43. Spinoza, Traité théologico-politique, cité ici dans la trad. franç. de Charles AppuHn, Paris, Garnier-Flammarion, 1965, p. 243-244.

44. Nous suivons ici la leçon de Y. Yovel, op. cit. supra n. 24, p. 194-195.

45. Ibid., p. 194.

46. SPINOZA, op. cit. supra n. 43 , p. 71. 
totalement infondée. D'une part, depuis Christianity not Mysterious (1696), Toland avait tâché de jeter les bases d'un christianisme épuré, dépouillé de ses mystères, et dont tout l'enseignement visât à susciter une obéissance raisonnable et un comportement authentiquement moral; d'autre part, Toland, qui a vécu en Hollande, a bel et bien lu et médité Spinoza, et, dans les articles de foi du Traité théologico-politique, il avait pu lire un credo minimal selon ses vœux. Pour n'être que rarement mentionné dans son œuvre, Spinoza a pourtant eu sur lui une influence majeure : que l'on songe aux deux dernières lettres à Serena, qui, sous couvert d'une réfutation de Spinoza, en constituent plutôt une interprétation matérialiste. Plus près encore du sujet qui nous occupe, on constate, et ce sera là la dernière justification de notre hypothèse, que la préface de Tetradymus contient un véritable démarquage du chapitre I du Traité théologico-politique (« De la Prophétie »), où Toland, évoquant le caractère hyperbolique du style de l'Ancien Testament, reprend les exemples mêmes qu'emploie Spinoza lorsqu'il traite des choses qui sont rapportées à Dieu « pour exprimer une chose au degré superlatif ${ }^{47}:$ les « cèdres de Dieu», les «montagnes de Dieu », un « sommeil de Dieu »; cependant que l'appel à l'instauration de la liberté de penser qui clôt, ou presque, Clidophorus, est comme un écho assourdi du chapitre xx du Traité de Spinoza («Où l'on montre que dans un État libre il est loisible à chacun de penser ce qu'il veut et de dire ce qu'il pense »).

Dans Clidophorus, Toland pose, et traite la question de la double philosophie, en suggérant que cette pratique antique est également répandue chez les modernes : or, il semblerait que, s'il est un philosophe auquel il pensait sans doute, et auquel Clidophorus rend un indirect hommage, c'est bien Spinoza, qui est, peut-être, la clé qu'annonçait le titre de l'ouvrage.

Pierre LuRBE (juin 1994).

47. SPINOZA, op. cit. supra n. 43 , p. 39. 